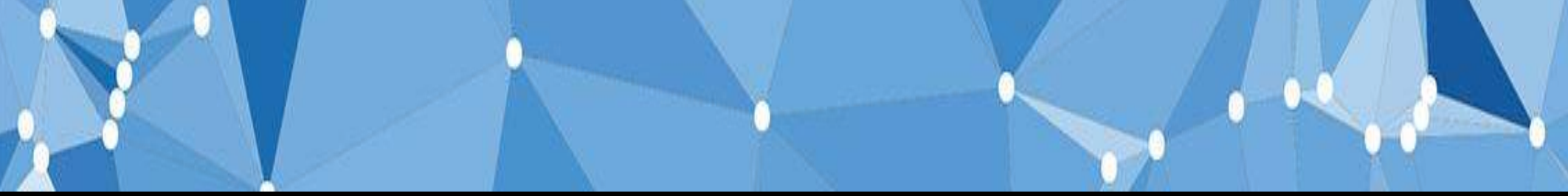

\title{
MOODSTRATIFICATION
}

\section{Immune Signatures for Therapy Stratification in Major Mood Disorder}

P.J. Goncalves Kling Lourenco', K. Bechter ${ }^{1}$, S. Claes $^{2}$, V. Arolt ${ }^{3}$, M. Leboyer ${ }^{4}$, F. Benedetti ${ }^{5}$, R. Furlan ${ }^{6}$, T. Munk-Olsen ${ }^{7}$, R. Berghmans ${ }^{8}$, B. Haarman ${ }^{9}$, R. Schoevers ${ }^{9}$.

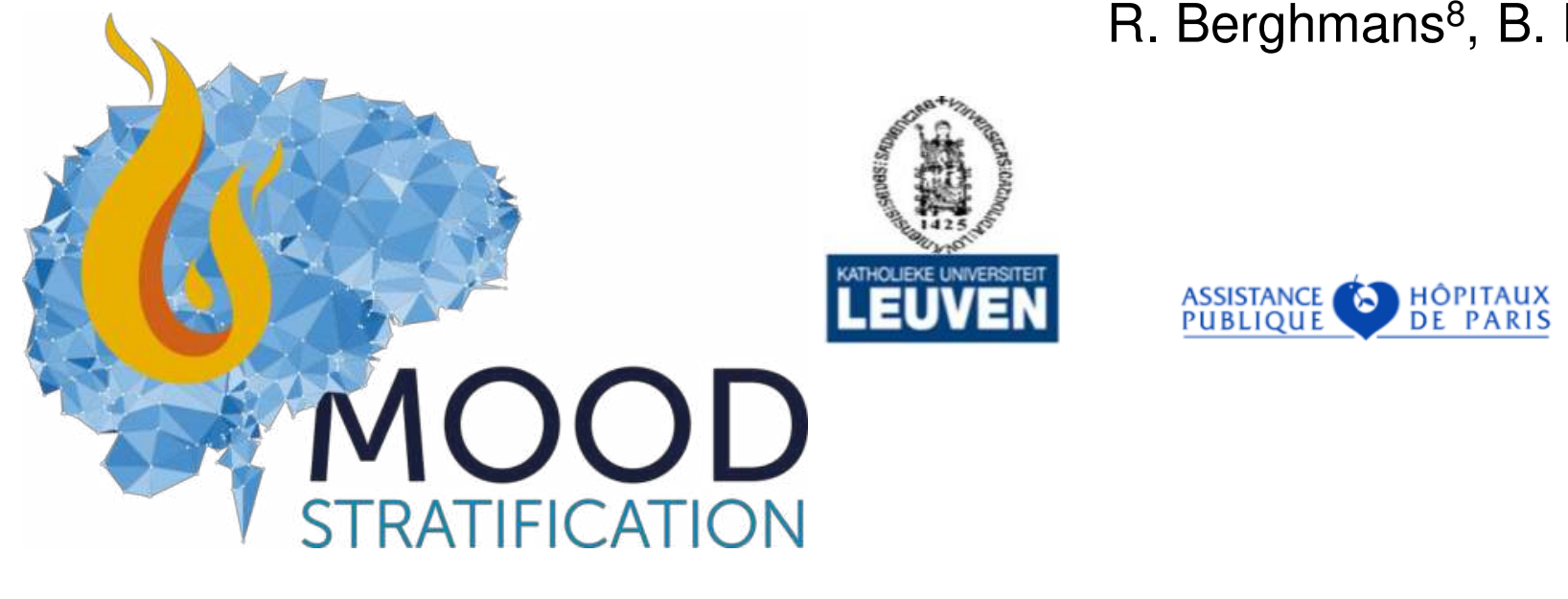

\section{Background/Hypothesis}

Previous research projects confirm that a large proportion of mood disorders are caused by the single action or the combination of Inborn and acquired $\mathrm{T}$ cell imbalances which result in both abnormal development and functioning of the brain, and lead to flares of chronic inflammation, this in combination with stress can lead to a high inflammatory state of the immune system. The aim of MOODSTRATIFICATION is to develop further evidence for this hypothesis and develop simple blood tests to measure the immune imbalances in clinical practice.

\section{Study Design}

MOODSTRATIFICATION is a large naturalistic, multi-center, cohort study, which aims to establish a proof-of-concept for a therapy stratification model of patients with mood disorders.

The study will be performed at 6 clinical centers and will include 390 patients of both genders and with at least a mild depressive episode (HDRS-17 score above 13). There will be two visits at the clinic (Baseline and week 8) and two online assessements at week 4 and 24. At Baseline a blood sample will be colected to determine each patient's immune profile and again at week 8 to investigate possible changes in the immune profiles.

\section{Objectives}

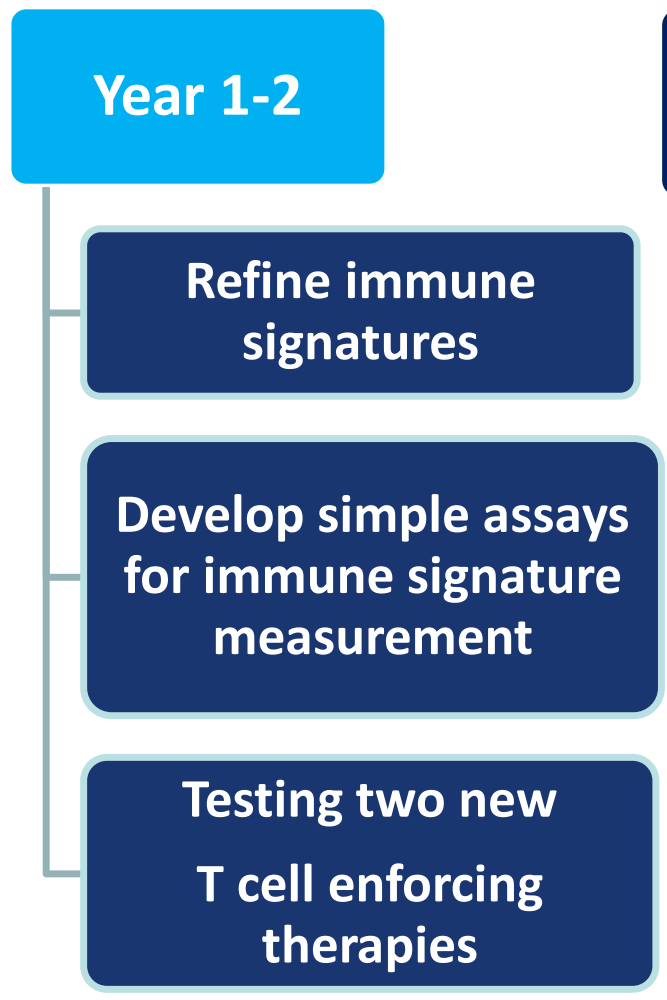

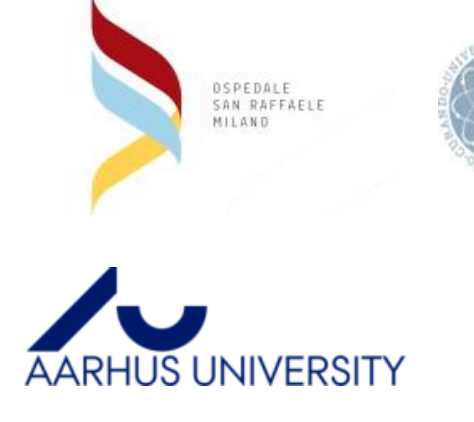
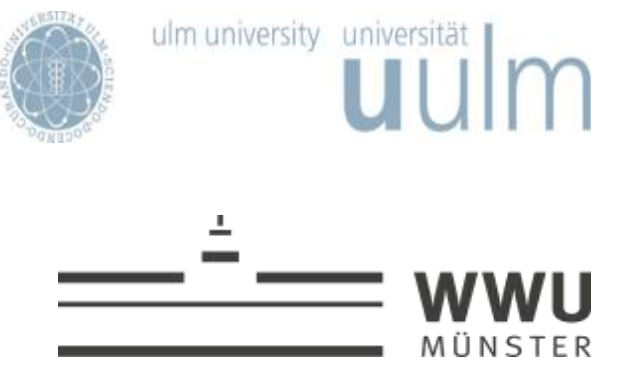

umcG

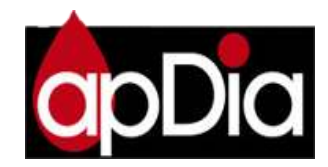

\section{Methods}

MOODSTRATIFICATION is divided in two phases. At phase one the patients will receive treatment as usual at the clinic. At this phase, regular care will be monitored and patients are allowed to receive additional psychosocial interventions. The primary outcome consists on the evaluation of patient treatment response after 8 weeks of regular treatment., based on the improvement of the HDRS-17 score across this period with $a \geq$ $50 \%$ decrease in HDRS-17 total score indicating full response and a $25-49 \%$ decrease in the total score indicating partial response. Secondary outcome consisting on changes in the IDS-R, BSS, and BAI total sum scores after 8 weeks treatment.

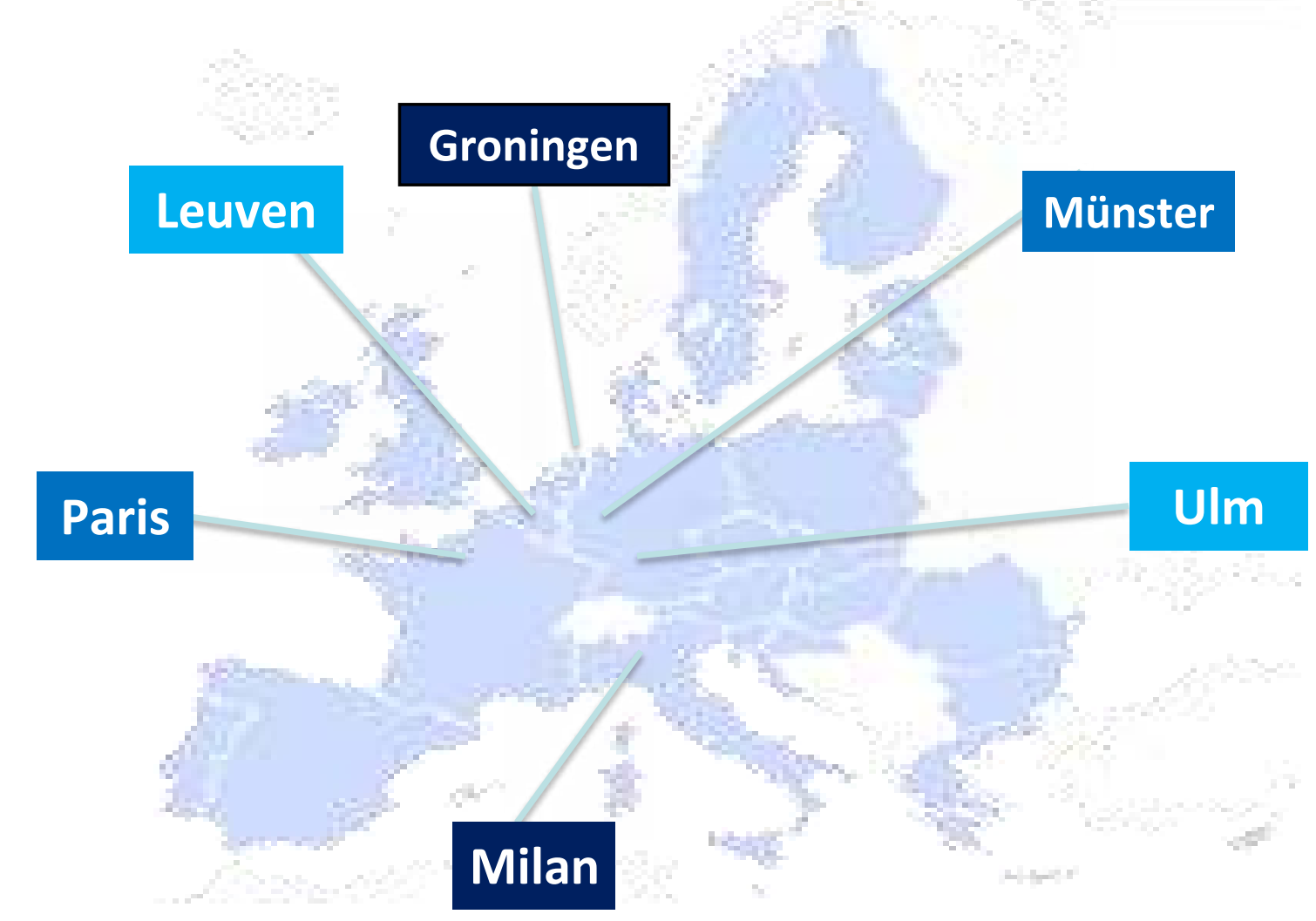

Mood Disorder Treatment Protocol

\begin{tabular}{|l|l|l|}
\hline 1 & - SSRI \\
\hline 2 & - Switch to other SSRI \\
\hline 3 & - SNRI \\
\hline 6 & - TCA \\
\hline
\end{tabular}

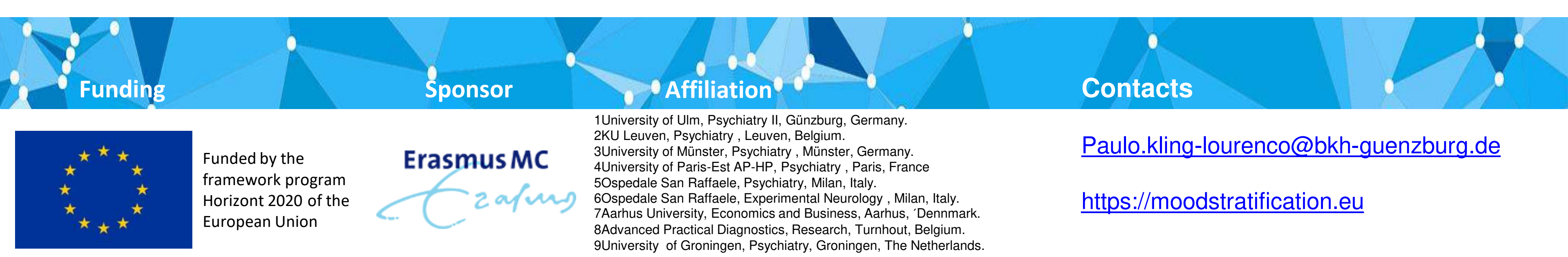

